\title{
Wind Inhomogeneities in [WC] Central Stars: From Late-to Early-Type Nuclei
}

\author{
A. Acker $^{1} \&$ Y. Grosdidier ${ }^{1,2}$ \\ ${ }^{1}$ Observatoire Astronomique de Strasbourg, Strasbourg, France \\ ${ }^{2}$ Instituto de Astrofísica de Canarias, La Laguna, Spain
}

In Grosdidier et al. $(2000,2001)$, wind fluctuations were described for five [WC 8-10] stars. In this poster we present new results discussing the case of the hotter subtype [WO 4] (Grosdidier \& Acker 2002). Specifically, we concentrate on the CIV $\lambda \lambda 5801 / 12$ emission-line variability observed for NGC 1501 and NGC 6751 (see also Acker \& Durand, these proceedings). Main results: NGC 1501: The OV $\lambda 5590$ and CIV $\lambda \lambda 5801 / 12$ emission lines as well as the CIV/CIII complex around $5690 \AA$ are variable at the $1 \%$ level. The amplitudes of the variations range from about $5 \%(\mathrm{OV})$, up to $7 \%$ (CIV) of the adjacent continuum flux. The HeI $\lambda 5876$ is also found to be variable; NGC 6751: For this star, significant variability at the $1 \%$ level is detected for the CIV $\lambda \lambda 5801 / 12$ emission line only. Note that the variations are quite huge since they span $6-10 \%$ of the adjacent continuum flux. Small variations are seen around the line centre but they are essentially located in the red and blue wings of the line, the latter showing the largest level of variability. Generally, the amplitudes of the variations in [WO 4] central stars range up to $10 \%$ of the adjacent continuum flux, over timescales of hours, or days. This result is essentially the same than that found for [WC]late type stars. We expect strong, hydrogen-deficient [WC] winds to be extreme examples for central stars of $\mathrm{PN}$, so that any fine structure found in [WC] winds may apply to all winds of central stars of $\mathrm{PN}$, much as one is finding now that weak, massive O-star winds also show the same fine structure as massive WR winds. The consequences of clumping in hot-star winds are manifold, including substantial constraints on the effective mass-loss rates, and their possible impact on the surrounding nebula itself (Acker et al. 2002). On the whole, the winds of all [WC] central stars are significantly stochastically variable on relatively short time-scales. This supports a turbulent origin.

\section{References}

Acker, A., Gesicki, K., Grosdidier, Y., Durand, S. 2002, A\&A, in press

Grosdidier Y., Acker A., Moffat A. F. J. 2000, A\&A 364, 597

Grosdidier Y., Acker A., Moffat A. F. J. 2001, A\&A 370, 513

Grosdidier Y., \& Acker A. 2002, A\&A, in preparation 